\title{
Non-surgical management of unruptured tubal ectopic pregnancy
}

\author{
Anjali Choudhary*, Priyanka Chaudhari, Neeta Bansal
}

Department of Obstetrics and Gynecology, Shree Guru Ram Rai Institute of Medical and Health Services, Patel Nagar, Dehradun, Uttarakhand, India

Received: 29 April 2017

Accepted: 29 May 2017

\section{* Correspondence:}

Dr. Anjali Choudhary,

E-mail: mcgsunil@gmail.com

Copyright: (c) the author(s), publisher and licensee Medip Academy. This is an open-access article distributed under the terms of the Creative Commons Attribution Non-Commercial License, which permits unrestricted non-commercial use, distribution, and reproduction in any medium, provided the original work is properly cited.

\begin{abstract}
Background: Ectopic pregnancy is still the leading cause of pregnancy related morbidity in the first trimester. Since majority of the women who present with ectopic pregnancies are sub fertile and young, there is a role for non-surgical options of managing these pregnancies. Expectant and medical management not only serves to conserve the fallopian tubes but also saves women from surgical trauma and morbidity. The objective of this retrospective study was to share our experience of treating un-ruptured tubal ectopic pregnancies conservatively.

Methods: Women diagnosed with un-ruptured tubal ectopic pregnancy, fit for conservative /medical management were included. Women with serum beta HCG levels less than $1000 \mathrm{mIU} / \mathrm{L}$ were treated expectantly and women with Bet HCG levels $>1000$ but $<10,000 \mathrm{mIU} / \mathrm{L}$ were given Injectable methotrixate. Response to treatment was monitored by serial beta HCG levels.

Results: Total 37 women included in the study.12\% women showed complete resolution with expectant treatment alone and $88 \%$ resolved after a single dose methotrixate.

Conclusions: Many women with un-ruptured tubal ectopic pregnancies would benefit from expectant management, or methotrixate therapy. Methotrixate used in carefully selected women is safe and effective in resolving these cases with good post treatment reproductive outcome.
\end{abstract}

Keywords: Conservative treatment, Ectopic pregnancy, Medical management

\section{INTRODUCTION}

Extra uterine implantation of an embryo is a flaw in the human reproduction. Ectopic pregnancy is still the leading cause of pregnancy related morbidity in the first trimester. Commonest site for ectopic pregnancy is the fallopian tubes. Over the years there has been a constant rise in the incidence of ectopic pregnancy, almost to the epidemic proportions. Overall incidence of ectopic pregnancy ranges from 1 in 161 to 1 in 80 pregnancies. One of the main causes of rise in the incidence is the early diagnosis of ectopic pregnancies. With the advent of trans-vaginal Ultrasonography and rapid quantitative beta HCG assays, since the 1980s, diagnosis has become easy and accurate. ${ }^{1}$ Many early extra uterine implantations of the embryos that were missed or misdiagnosed as delayed menses, have also been brought under the diagnostic scanner. Due to a high index of suspicion many ectopic gestations are diagnosed in early stages before the rupture. Wide spread use of medical abortions, MTPs, incompletely treated post abortion or puerperal sepsis, STDs, use of emergency contraception, previous history of an ectopic pregnancy are a few causes incriminated in the rising trend. ${ }^{1,2}$ Main cause of tubal ectopic pregnancy is tubal dysfunction, either anatomic or functional, due to infections, previous surgeries or alteration in tubal motility, as caused by certain post coital contraception containing high dose levonogestrel. 
Alteration and inhibition of tubal motility, reduced fimbrial beating leading to delayed arrival of an embryo in the endometrial cavity is believed to be the mechanism by which progesterone only post coital pills are suggested to cause ectopic pregnancy. Pregnancies occurring in women using daily progestogen-only oral contraceptive pills are more likely to be ectopic than pregnancies in users of other contraceptive methods, so when women on POP with amenorrhea have positive pregnancy test, an ectopic pregnancy should be considered..$^{3-5}$

Since majority of the women who present with ectopic pregnancies are sub fertile and young, there is a role for non-surgical options of managing these pregnancies. Expectant and medical management not only serves to conserve the fallopian tubes but also saves women from surgical trauma and morbidity. Medical management of ectopic pregnancies with methotrexate an antimetabolite drug has been proven to be safe and effective. ${ }^{6}$ Proper selection of cases fit for medical management improves the success rate. Many of the tubal ectopic gestation resolve with time even without treatment and some will benefit from treatment with anti-metabolite drugs.

Methotrexate (MTX) is an antimetabolite chemotherapeutic agent which acts by interfering in DNA synthesis of rapidly growing cancer cells. Same property can be used to halt the growth of a developing embryo. Methotrexate has been effectively used in treatment of leukemias, lymphomas, carcinomas of head neck, ovary and breast. It has been successfully used in managing molar pregnancies and chorio-carcinoma. As used in the treatment of ectopic pregnancy, methotrexate is administered in a single or in multiple intramuscular (IM) injections.

Successful medical treatment using methotrexate has been reported in the literature with good subsequent reproductive outcomes. By avoiding surgery, the risk of tubal injury can be reduced

Objective: objective of this retrospective study is to share our experience of treating unruptured tubal ectopic pregnancies conservatively.

\section{METHODS}

Women diagnosed with un-ruptured tubal ectopic pregnancy, fit for conservative/medical management were included.

Diagnosis was made on the basis of history of amenorrhea, a positive pregnancy test and ultrasonographic findings of an empty uterus and/or an adnexal mass or gestational sac.

\section{Inclusion criteria}

Stable patient, un-rupture tubal pregnancy, gestation sac less than three to four $\mathrm{cm}(3.5 \mathrm{~cm}$ without cardiac activity), absence of hemoperitoneum, absence of cardiac activity, baseline beta HCG less than 10,000, patient's willingness to avail medical management and availability for follow up.

\section{Exclusion criteria}

Unstable women, presence of hemoperitoneum, larger sacs with live fetal pole, serum beta HCG more than 10,000. Consent was taken for conservative/medical management with the understanding of the need for surgical option in the advent of a failed treatment or rupture. Pretreatment counseling was done.

Pretreatment investigations included serum beta HCG levels, Complete blood count, Platelet counts, kidney and liver function tests. Measurement of height and weight to calculate body surface area was done.

After admission and initial work up women with baseline beta HCG of less than 1000 were treated conservatively by monitoring vital signs, beta HCG was repeated every 48 hours to check the falling trend of a failing ectopic gestation.

Another group of women with unruptured ectopic gestations who met the criteria, having baseline beta HCG more than 1000 but less than 5,000 were given injection Methotrexate $(50 \mathrm{mg} / \mathrm{sq}$. meter of body surface area). Beta HCG titers were repeated after 48 hours. A 15 $\%$ fall of beta HCG was taken as successful treatment and further treatment included observation of falling beta HCG levels. In the absence of an appreciable fall or rise of beta HCG levels, a repeat dose of methotrexate was given after testing the total and differential counts.

The fall of Beta HCG less than 25mIU/L without surgical intervention was considered successful medical treatment. Patients were advised to avoid vitamins containing folic acid, NSAIDs, alcohol intake, exposure to sun after treatment with methotrexate. Growth of pregnancy or rupture of ectopic sac requiring surgery was taken as a failure of the treatment. Course of treatment and outcomes were tabulated and analyzed.

\section{RESULTS}

A total of 37 cases diagnosed with unruptured tubal ectopic pregnancies were studied retrospectively over a course of one year.

Ten women with initial serum beta HCG less than 1000 $\mathrm{mIU} / \mathrm{L}$ were given expectatnt treatment. All ten women showed downward trend of serum B HCG and resolved completely.

Twenty-three women with pretreatment Beta HCG levels more than 1000 but less than 10,000 mIU/L received injection methotrexate. Out of this group 18 woman had resolution and showed downward trend of beta HCG on 
Post treatment evaluation. Four patients showed continued rise in b HCG and needed second dose of methotrexate. Three patients showed continued growth of pregnancy and needed surgical treatment. Two patients had tubal rupture and needed emergency surgery. Mean gestational age at diagnosis was 6.4 weeks.

Mean pretreatment serum Beta HCG was 552.54 IU/L in the group of women who received expectant treatment and 5175.64 IU/L in patients who received methotrexate injection. Age and gravidity distribution of patients is as given in the tables. All the women in the expectant group showed complete resolution.

In the group with methotrexate therapy success rate was $88 \%$ and $12 \%$ women required surgical treatment, due to rupture and/or rising trend of beta HCG. Two patients developed neutropenia after methotrexate and was given
Injection filgrastin. Most patients tolerated treatment well with no major side effects.

Table 1: Distribution of women according to parity.

\begin{tabular}{|lll|}
\hline Gravida & Number & $\%$ \\
\hline G1 & 6 & 16.21 \\
\hline G2 & 15 & 40.54 \\
\hline G3 & 11 & 29.7 \\
\hline G4 & 5 & 13 \\
\hline
\end{tabular}

Table 2: Distribution of women according to age.

\begin{tabular}{|lll|}
\hline Age group & Number & $\%$ \\
\hline $20-25$ years & 11 & 29.7 \\
\hline $26-30$ years & 16 & 43.24 \\
\hline $31-35$ years & 10 & 27.02 \\
\hline $35-40$ years & - & \\
\hline
\end{tabular}

Table 3: Treatment outcome.

\begin{tabular}{|llllll|}
\hline No. of patients & Type of treatment & $\begin{array}{l}\text { Outcome } \\
\text { Resolution }\end{array}$ & Required second dose & Ruptured & Required surgery \\
\hline $27(81 \%)$ & Methotrexate & $18(78 \%)$ & $4(17.39 \%)$ & $2(8.69 \%)$ & $3(13.04 \%)$ \\
\hline $10(27 \%)$ & Expectant & $10(100 \%)$ & & & \\
\hline
\end{tabular}

Mean pre-treatment Beta HCG level: Expectant group-552.54 IU/L; Methotrexate group- 5175.64IU/L;

\section{DISCUSSION}

Ectopic pregnancy and its outcome is a major contributor to maternal morbidity and mortality. Economic burden and impairment of fertility is a great concern. Among the greatest advances in the management of ectopic pregnancy has been the development of medical management, which became available in the mid-1980s. ${ }^{1}$ Initial protocols for medical therapy required long-term hospitalization and multiple doses of methotrexate and were associated with significant side effects. Modification and refinement of these protocols, however, have led to single-dose outpatient therapy. ${ }^{6,7}$

There has been some debate on how to choose between medical and surgical treatment for an un-ruptured tubal ectopic pregnancy. According to the guidelines formulated after analyzing the Cochrane data base trials registry it is safe to presume that surgical treatment should be opted if patient is hemodynamically unstable, serum hCG levels are $>10,000 \mathrm{mIU} / \mathrm{L}$, the sac size is $>/=$ $4 \mathrm{~cm}$ and there is a medical contraindication to MTX use. Medical treatment can be considered when serum HCG levels are below $5000 \mathrm{mIU} / \mathrm{L}$ or $10,000 \mathrm{mIU} / \mathrm{L}$, the sac size is less than $4 \mathrm{~cm}$ and hemodynamically stable patient. Medical treatment should be preferred if s- hCG levels are less than $1000 \mathrm{mIU} / \mathrm{L}$ patient has no pain and ectopic sac is not visualized on $\mathrm{USG}^{4}$. We followed the same guidelines in our selection of patients. There is no consensus whether the upper limit of initial S, HCG levels should be less than 5000 , or $10,000 \mathrm{mIU} / \mathrm{L}$, for initiation of MTX therapy, different authors follow different criteria. The rate of success of treatment of course depends upon the pretreatment HCG levels. ${ }^{8}$ According to study of ectopic pregnancies by Mahi Ak et al. Expectant management and medical therapy was offered only in $1.2 \%$ and $1.75 \%$ respectively. ${ }^{2}$ Modern management of un-ruptured tubal ectopic pregnancies primarily focuses on non-surgical treatment. In united states the percentage of women treated with methotrexate has risen from $11.1 \%$ to $35 \%$, whereas the surgical management has fallen from $90 \%$ to $65 \%$. Many earlier studies on safety and efficacy of methotrexate have been conducted with an overall success rate of $92 \%$.

A study on small un-ruptured ectopic pregnancies with pretreatment beta HCG levels less than $5000 \mathrm{mIU} / \mathrm{L}$ reported a successful outcome in $85 \%$ of cases and observed that women with initial beta HCG levels of $3000-4000 \mathrm{mIU} / \mathrm{L}$ required a second dose or surgical treatment. ${ }^{8}$ In present study it was observed that a success rate of $88 \%$ with a single dose therapy, $4 \%$ women required a second dose and $8 \%$ needed surgical intervention. Alshimmiri MM et al in a similar study with single dose MTX quoted a $95 \%$ success rate when mean pretreatment beta HCG level was $2592 \mathrm{mIU} / \mathrm{L} .{ }^{8}$ A single dose therapy is safe, easily tolerated, associated with minimal cost when used in carefully selected patients. ${ }^{9}$ 
There is group of women with ectopic gestation with low pretreatment serum hCG levels (below 1000) which would undergo spontaneous resolution even without treatment, and only need vigilance, we found $27.2 \%$ of women in this group. Various regimen for use of methotrexate in medical management of ectopic pregnancy have been tried. Barthart et al in a metaanalysis compared single dose and multi-dose regime and concluded that multi-dose regimes are more effective. ${ }^{6}$ Although in a later study Guvendag Guven found that single dose therapy is equally effective and that multiple doses might increase the risk of side effects of methotrexate. A multi-dose therapy when used helps to bring down hCG levels to less than $25 \mathrm{mIU} / \mathrm{L}$ faster than a single dose. $^{6}$ Soliman $\mathrm{KB}$ et al found single intramuscular dose sufficient to manage small ectopic pregnancies, and concluded that success rate depends upon initial s.hCG levels. ${ }^{10}$ In study a single dose of MTx was used and observed resolution of in $88 \%$ of ectopic pregnancies. It was easily tolerated; there were only minor side effects. Overall success rate in managing unruptured tubal ectopic gestation without surgery in our experience was $90 \% .^{11}$

\section{CONCLUSION}

Considering the type of women who present with ectopic pregnancies, who are young and sub fertile, there a role for conservative management of these pregnancies. Nonsurgical management not only serves to conserve fallopian tubes but also avoids the morbidity of surgical intervention. Many women would benefit from expectant management, or methotrexate therapy. Methotrexate used in carefully selected women is safe and effective in resolving these cases with good post treatment reproductive outcome.

\section{ACKNOWLEDGMENTS}

Authors would like to thank for the support of the management and the Obstetrics and gynecology department.

Funding: No funding sources Conflict of interest: None declared

Ethical approval: The study was approved by the Institutional Ethics Committee

\section{REFERENCES}

1. Chi TJ, Dyne PL. Emergent Management of Ectopic Pregnancy. Available http://emedicine.medscape.com/article/796451overview.

2. Majhi AK, Roy N, Karmakar KS, Banerjee PK. Ectopic pregnancy--an analysis of 180 cases. Journal of the Indian Medical Association. 2007;105(6):30810.

3. Harrison-Woolrych M, Woolley J. Progestogen-only emergency contraception and ectopic pregnancy [editorial]. J Fam Plann Reprod Health Care. 2003;29(1):5-6.

4. Canis M, Savary D, Pouly JL, Wattiez A, Mage G. Ectopic pregnancy: criteria to decide between medical and conservative surgical treatment? J Gynecol Obstet Biol Reprod (Paris). 2003;32(7Suppl):S54-63.

5. Pereira PP, Cabar FR, Raiza LC, Roncaglia MT, Zugaib M. Emergency contraception and ectopic pregnancy: report of 2 cases. Clinics. 2005;60(6):497-500.

6. Barnhart KT, Gosman G, Ashby R, Sammel M The medical management of ectopic pregnancy: a metaanalysis comparing single dose and multidose regimens. Obstet Gynecol. 2003;101(4):778-84.

7. Guvendag Guven ES, Dilbaz S, Dilbaz B, Aykan Yildirim B, Akdag D, Haberal A. Comparison of single and multiple dose methotrexate therapy for unruptured tubal ectopic pregnancy: a prospective randomized study. Acta obstetricia et gynecologica Scandinavica. 2010;89(7):889-95.

8. Alshimmiri MM, Al-Saleh EA, Al-Harmi JA, AlSalili MB, Adwani AA, Ibrahim ME. Treatment of ectopic pregnancy with a single intramuscular dose of methotrexate. Arch Gynecol Obstet. 2003;268(3):181-3.

9. Slaughter JL, Grimes DA. Methotrexate therapy. Nonsurgical management of ectopic pregnancy West J Med. 1995;162(3):225-8. PMCID: PMC1022704

10. Soliman KB, Saleh NM, Omran AA. Safety and efficacy of systemic methotrexate in the treatment of unruptured tubal pregnancy. Saudi Med J. 2006;27(7):1005-10.

11. Arora P. Ectopic pregnancy after progesterone only emergency contraception, contraception for women: an evidence based overview. BMJ ed-letter to editor. 2013.

Cite this article as: Choudhary A, Chaudhari $\mathrm{P}$, Bansal N. Non-surgical management of unruptured tubal ectopic pregnancy. Int J Reprod Contracept Obstet Gynecol 2017;6:3041-4. 